five patients by Alberts et al. ${ }^{4}$ In all but one of their patients other associated neurological findings were present, indicative of widespread meningeal involvement by the time of cochlear manifestation. Neuro-otological testing performed on three patients disclosed bilateral caloric weakness in two. However, neither of these patients was reported to have vestibular symptoms. Other authors have described ataxia and hearing loss as the presenting symptom of carcinomatous meningitis. ${ }^{5}$ Nevertheless, in most of the reports, no conclusions were made as to whether the ataxia was due to hydrocephalus, cerebellar carcinomatous involvement, spinal cord compression, metastatic destruction of the pyramidal bone, or if it was the result of vestibular nerve infiltration. Moreover, neuro-otological testing was usually not performed.

The spread of neoplastic cells to the meninges occurs either directly from an adjacent tumour, or from distant primary tumours by the blood stream, or perivascular or perivenous lymphatics. ${ }^{5}$ Pathological examination shows that neoplastic cells extend in the temporal bone as far as the geniculate ganglion, where the subarachnoid space terminates, but can penetrate the cribriform area of the labyrinth. Labyrinthine metastasis of the tumour is a common finding, whereas selective internal auditory canal infiltration, as in our patient, is rare ${ }^{5}$.

The proximity of the VIIth and VIIIth nerves in the internal auditory canal makes both of them vulnerable to pathological changes in this location. Indeed, from the 15 patients with carcinomatous meningitis and labyrinthine involvement reported by Oshiro et al, unilateral or bilateral facial nerve palsy was documented in nine $\mathrm{e}^{5}$ Our patient had an old facial palsy after viral infection 10 years previously. Recent damage, such as by neoplastic cell involvement, would have probably abolished the facial synkinesiasevidence of a long standing, partly regenerated nerve lesion.

The syndrome of bilateral vestibular failure consists of gait unsteadiness and oscillopsia. The severe instability of stance in our patient might have been due to a polyneuropathy induced by the cancer or chemotherapy, even though gross proprioception defects were not detected on clinical testing. Our patient did not complain about oscillopsia or other visual phenomena attributable to vestibular failure. As patients with bilateral vestibular failure have impaired perception of motion, oscillopsia is perceived less than the real retinal image slip and $30 \%-40 \%$ of patients with bilateral vestibular failure never complain about this visual phenomenon. ${ }^{16}$

Infectious meningitis is not rare among the multiple causes of bilateral vestibular failure. ${ }^{12}$ However, carcinomatous meningitis is encountered much less and here bilateral vestibular failure is usually a part of a syndrome of meningeal infiltration with multiple cranial and spinal nerve involvement, hydrocephalus, or cerebral changes. ${ }^{3-5}$ In our patient the vestibulocochlear nerves were selectively involved. The only other symptom suggestive of meningitis was her complaint about a diffuse headache. Although patients with vestibular lesions often report headache, this is usually located in the neck and probably results from disturbed vestibulocollic reflexes. ${ }^{1}$

Carcinomatous meningitis is encountered in the course of metastatic breast cancer in
$1 \%-3.5 \%$ of patients. ${ }^{3}$ Intrathecal administration of methotrexate, radiotherapy, or the combination of both, are today the treatment options, but the optimal treatment has not been established and is individual for each patient. Despite aggressive therapy, most patients die within 6 months of diagnosis, the median survival being $6-16$ weeks. $^{7}$ It has been reported that response after 2 weeks of therapy is correlated with survival. ${ }^{7}$ Unfortunately, our patient did not respond to therapy.

L POLLAK

R MILO

V KOSSYCH

M J RABEY

Department of Neurology, Assaf Harofeh Medical Center, affiliated to Sackler Faculty of Medicine, Tel-Aviv University, Israel

E SHAPIRA

Department of Oncology

Correspondence to: Dr L Pollak, 1 Neve-Nir, NesZiona 74 042, Israel

1 Brandt T. Bilateral vestibulopathy. In: Brandt T, editor. Vertigo. Its multisensory syndromes, 2 nd edition. London: Springer Verlag, 1999:127-41.

2 Rinne T, Bronstein AM, Rudge P, et al. Bilateral

Rinne T, Bronstein AM, Rudge P, et al. Bilateral
loss of vestibular function. Acta Otol Suppl loss of vestibular

3 Jayson GC, Howell A, Harris M, et al. Carcinomatous meningitis in patients with breast cancer. Cancer 1994;74:3135-41.

4 Alberts MC, Terrence CF. Hearing loss in carcinomatous meningitis. I Laryngol Otol 1978;92:233-41.

5 Oshiro H, Perlman HB. Subarachnoid spread of tumor to the labyrinth. Arch Otolaryngol 1965; 81:328-34.

6 Grunfeld EA, Morland AB, Bronstein AM, et al. Adaptation to oscillopsia. A psychophysical Adaptation to oscillopsia. A psychophysical 123:277-90.

7 Grant R, Naylor B, Greenberg HS, et al. Clinical outcome in aggressively treated meningeal carcinomatosis. Arch Neurol 1994;51:457-61.

\section{Loss of silent reading in frontotemporal} dementia: unmasking the inner speech

Presenting signs of frontotemporal dementia usually include social disinhibition, loss of initiative, compulsive features, cognitive decline, and motor symptoms. Behavioural features have recently been detailed, in an attempt to distinguish patients with frontotemporal dementia from patients with Alzheimer's disease. ${ }^{12}$ We report a new feature inaugurating this syndrome which could be a strong illustration of a prefrontal inhibitory role in the control of inner speech, through an early loss of silent reading as an inaugural sign of frontotemporal dementia.

A 69 year old man, a retired baker, with no family history of neurological disease, was admitted to the neurological department for evaluation and diagnosis of a progressive dementia with movement disorders. Since the beginning of 1998 (when he was 67), his wife had noticed that he had slight head movements with small amplitude, occurring only while watching TV. During 1998 the patient had started to read aloud at bedtime, annoying his wife. His voice was very loud, and it was difficult for him to stop. Moreover, he often spoke aloud to himself during the day or suddenly began singing, all features which were very unusual for him. Later in the same year, he showed memory impairment, loss of motivation, and frequent throat clearing sounds. In 1999, a gait disorder with occasional falls appeared, while stereotypic movements resembling temple rubbing with both hands developed. At this time, the patient presented with severe depressive symptoms and became bedridden. At examination, in March 2000, he was able to stop motor and vocal stereotypies for up to 1 minute. After discontinuing the self control, a rebound effect occurred and the patient showed repetitive preauricular rubbing with both hands, with an associated meaningless vocalisation. When asked why he performed these movements, he answered that it was a habit he had developed before. Severe dysarthria and drooling, with swallowing dysfunction were present, but no motor deficit or sensory loss was evidence. Bilateral grasping phenomena, perserveration, and imitative behaviour were easily elicited. Slight symmetric distal akinesia with minimal rigidity was present, but the patient was taking antidopaminergic agents. There were no cerebellar, pyramidal, or dysautonomy signs, or oculomotor palsy. Extensive investigations were negative (including blood sample analysis, genetic testing for Huntington's disease, EEG, brain MRI, and cutaneous biopsy). Neuropsychological tests confirmed the dysexecutive syndrome, with a Mattis score of $122 / 144$ in July 1999, dropping to 87 in March 2000. In August 2000, a percutaneous endoscopic gastrostomy was performed due to swallowing difficulties and a substantial loss of weight. At this time, he continuously repeated his compulsive motor stereotypies and utterances, mainly cursing. Treatment with tetrabenazine, flupentixol, and hydroxyzine were of little help. He died in October 2000 and there was no postmortem analysis.

The patient reported here presented with FTD, according to the current criteria. ${ }^{3} \mathrm{He}$ progressively developed behavioural abnormalities including motor and verbal stereotypies, coprolalia, with poor self control, although he remained able to discontinue them momentarily. He performed very poorly on the frontal battery test and failed to improve under various drug regimens. Brain MRI remained generally atrophic. We suggest that the pathological process underlying his progressive loss of behavioural control was already at work when the patient became unable to read silently. Inner speech is an understudied phenomenon giving rise to mentation under internal control. The major role of "speech for self" in the developing human has been outlined. ${ }^{4}$ Silent reading could be an externally triggered inner speech as opposed to an internal production of inner speech linked to the thinking process. Besides the generating mechanisms producing inner speech at the interface of thought and language, an endogenous control must exist to inhibit the utterance of inner speech. A pathological process involving this inhibitory control may unmask inner speech. We speculate that such an inhibitory control of inner speech may be pathologically involved in our patient, underlying both initial loss of silent reading, and the most recent vocal compulsive stereotypies. Prefrontal cortical dysfunction may be the underlying pathophysiological mechanism explaining the inability to inhibit the vocalisation of inner speech in our patient, as assumed by the final clinical diagnosis.

L VERCUEIL H KLINGER Neurological Department, Grenoble University Hospital, 38043 Grenoble cedex 9, France

Correspondence to: $\mathrm{Mr} \mathrm{L}$ Vercueil

vercueil@lycos.com

1 Bozeat S, Gregory CA, Ralph MA, et al. Which neuropsychiatric and behavioural features distinguish frontal and temporal variants of 
frontotemporal dementia from Alzheimer's disease? If Neurol Neurosurg Psychiatry 2000;69:178-86.

2 Levy ML, Miller BL, Cummings JL, et al. Alzheimer disease and frontotemporal dementias: behavioral distinctions. Arch Neurol 1996;53:687-90.

3 Neary D, Snowden JS, Gustafson L, et al. Frontotemporal lobar degeneration: a consensus on clinical diagnostic criteria. Neurology 1998;51: $1546-52$.

4 Kinsbourne $M$. Inner speech and the inner life. Brain Lang 2000;71:120-3.

Anti-Jo-1 positive inclusion body myositis with a marked and sustained clinical improvement after oral prednisone

The three major categories of the idiopathic inflammatory myopathies are dermatomyositis, polymyositis, and inclusion body myositis. ${ }^{1}$ The most important clinical feature distinguishing inclusion body myositis from dermatomyositis and polymyositis is the lack of responsiveness to immunosuppressive treatment. ${ }^{12}$ Yet, it is the experience of many clinicians that a small subgroup of patients with inclusion body myositis show at least a partial response to immunosuppressive treatment. There are no specific characteristics which can identify this subgroup. In this report we present an anti-Jo-1 positive patien with inclusion body myositis who showed a marked and sustained clinical improvement after treatment with oral prednisone.

A 74 year old man, with no relevant medical history, presented with a slowly progressive proximal muscle weakness of the lower limbs. On presentation there were no complaints of muscle weakness of the upper limbs, dysphagia, myalgia, arthralgia, or feelings of general malaise. He denied any sensory symptoms. His family history was negative for neuromuscular or rheumatic disorders and he did not use any myotoxic drugs.

Physical examination showed asymmetric proximal and distal muscle weakness (muscle strength in MRC grades: neck flexors 3, right and left triceps 4 , right and left iliopsoas 4 , right and left gluteus maximus 4 , right quadriceps 4 , left quadriceps 2.5 , right and left hamstrings 4 , right and left anterior tibial 2 , left gastrocnemius 4, all other muscles 5), marked atrophy of the quadriceps muscles and low symmetric tendon reflexes. The muscles were not painful to palpation. All other aspects of the general and neurological examination were normal.

Laboratory investigations showed slightly increased concentrations of serum creatine kinase $(260 \mathrm{U} / 1 ;$ normal<200 U/1). All other aspects of the routine laboratory investigation including erythrocyte sedimentation rate, lactate dehydrogenase, vitamins, thyroid function tests, and antinuclear factor were normal or negative. Serum was screened for the presence of myositis specific autoantibodies (MSAs) using immunoblotting, enzymelinked immunosorbent assay (ELISA), and immunoprecipitation as previously described. ${ }^{3}$ Assays were positive for the antiJo-1 autoantibody.

Electromyography demonstrated fibrillation potentials with positive sharp waves, polyphasia, short duration small amplitude motor unit potentials, and several high amplitude motor unit potentials in proximal and distal muscles. Nerve conduction studies were normal.

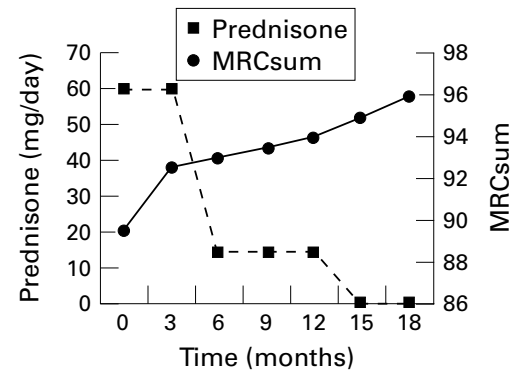

diagnosis between the three entities by virtually excluding inclusion body myositis in cases of anti-Jo-1 positivity and therefore providing an additional argument for the start of immunosuppressive therapy. ${ }^{4}$

The clinical and electrophysiological improvement after immunosuppressive therapy in the presented patient, together with the presence of a disease specific autoantibody, suggests a prominent role of the inflammatory response. Although it is only based on one case history, this report raises the question whether the presence of an MSA can aid in the identification of patients with inclusion body myositis who might show a response to immunosuppressive therapy. It is not known how MSAs are generated and whether they represent an epiphenomenon or whether they are somehow involved in the pathogenesis of idiopathic inflammatory myopathies. Based on their specificity for myositis it does seem likely that they are the result of a yet unidentified immunological mechanism which is specific for idiopathic inflammatory myopathies and which is, directly or indirectly, linked to the occurrence of clinical myopathy. It can be hypothesised that the presence of an MSA in inclusion body myositis is the result of an identical immunological mechanism as in MSA positive patients with dermatomyositis or polymyositis and that thus immunosuppressive treatment would be of benefit, as in this patient. Additional studies are required to consider these questions.

In conclusion, the present data suggest that in a patient with an idiopathic inflammatory myopathy, even inclusion body myositis, and the presence of an MSA, immunosuppressive treatment should be started and continued for at least 3 months.

We thank WTM Vree Egberts, BAW de Jong, and E Nuy-Terwindt for their expert technical assistance. This work was supported by grant 93-1112 of the Prinses Beatrix Fonds and grant 940-37-009 of MW-NWO.

G J D HENGSTMAN $\begin{array}{lr}\text { small amplitude motor unit potentials. Pred- } & \text { H J TER LAAK } \\ \text { nisone was slowly tapered and stopped 1 year } & \text { B G M VAN ENGELEN }\end{array}$ $\begin{array}{lr}\text { small amplitude motor unit potentials. Pred- } & \text { H J TER LAAK } \\ \text { nisone was slowly tapered and stopped 1 year } & \text { B G M VAN ENGELEN }\end{array}$ after the initiation of treatment. strength remained stable (muscle strength in MRC grades 18 months after treatment initiation: right and left triceps 4, right and left quadriceps 4 , right and left hamstrings 4 , right and left anterior tibial 2, all other muscles 5).

What makes this particular case of great interest are the presence of the anti-Jo-1 autoantibody and the marked and sustained clinical improvement after treatment with oral prednisone. Although a few studies have reported mild improvement in inclusion body myositis with various immunomodulating agents, significant and sustained improvement remains extremely rare in inclusion body myositis. ${ }^{12}$

The anti-Jo-1 autoantibody is the most prevalent MSA and is found in $25 \%$ of patients with dermatomyositis and patients with polymyositis. ${ }^{4}$ In patients with inclusion body myositis the antibody is hardly ever detected. ${ }^{4}{ }^{5}$ Until now, only three patients with inclusion body myositis have been reported in whom the antibody was found. ${ }^{5}$ Unfortunately, the clinical picture of these patients was not described. The relative absence of Jo-1 in inclusion body myositis is seen as support for the hypothesis that the immune response in this disease differs from that in dermatomyositis and polymyositis. ${ }^{4} \mathrm{It}$ has therefore been suggested that the antiJo-1 autoantibody can aid in the differential
Neuromuscular Centre Nijmegen, Institute of Box 9101, 6500 HB Nijmegen, The Netherlands
Bijology, University Medical Centre Nimegen, PO W J VAN VENROOIJ

Department of Biochemistry, University of Nijmegen, Nijmegen, The Netherlands

Correspondence to: Dr B G M van Engelen

b.vanengelen@czzoneu.azn.nl

1 Dalakas MC. Polymyositis, dermatomyositis and inclusion-body myositis. $N \mathrm{Engl} \mathcal{F} \mathrm{Med}$ 1991;325:1487-98 sion body myositis and myopathies. Ann Neurol 1995;38:705-13.

3 Rutjes SA, Vree Egberts WT, Jongen P, et al. Anti-Ro52 antibodies frequently co-occur with anti-Jo-1 antibodies in sera from patients with idiopathic inflammatory myopathy. Clin Exp Idiopathic inflammatory

4 Hengstman GJ, van Engelen BG, Badrising UA, et al. Presence of the anti-Jo-1 autoantibody excludes inclusion body myositis. Ann Neurol 1998;44:423.

5 Koffman BM, Rugiero M, Dalakas MC. Immune-mediated conditions and antibodies associated with sporadic inclusion body myositis. Muscle Nerve 1998;21:115-17.

\section{Upholsterer's PIN}

Posterior interosseous nerve (PIN) palsy has been recognised at least since 1905 when Guillain and Courtellemont described a case in an orchestral conductor. In 1899 Gowers described involvement of the muscles of the
2 Griggs RC, Askanas V, DiMauro S, et al. Inclu- 